\title{
Multidimensional-signal sample dependency at Nyquist densities
}

\author{
Robert J. Marks II \\ Department of Electrical Engineering, FT-10, University of Washington, Seattle, Washington 98195
}

Received July 2, 1984; accepted September 23, 1985

\begin{abstract}
When a multidimensional signal is uniformly sampled, its spectrum is replicated. If the signal is band limited and the replications (1) contain regions that are identically zero and (2) are not aliased, then the samples are dependent. Indeed, lost samples can be regained from those remaining. In dimensions greater than one, there are spectral regions of support for which this is the case even when sampling is performed at the Nyquist (minimum) density (e.g., a circular spectral region of support in two dimensions). When the known samples are perturbed by additive noise, lost-sample restoration noise levels in certain cases can be obtained by simple geometrical observations in the frequency domain. The results are specifically applied to coherent and incoherent optical images of objects of finite extent obtained from imaging systems with circular pupils.
\end{abstract}

\section{INTRODUCTION}

In one dimension, a band-limited signal's samples are independent when sampling is performed at the Nyquist rate. In higher dimensions, band-limited signal samples obtained at Nyquist (minimum) densities can display a strong dependence. Indeed, lost samples can be regained from those remaining. In the one-dimensional case, oversampling is required for sample dependency. 1,2

The ability to restore lost samples of a multidimensional band-limited signal sampled at Nyquist density is determined solely by the shape of the support of the signal's spectrum. If the shape is such that replicated nonoverlapping versions can fill the space with no gaps, then Nyquist samples are independent. Otherwise, they are not.

An example of the former in two dimensions is a rectangle. A circle is an example of the latter. Any coherent or incoherent image of an object of finite extent obtained from an imaging system with a circular pupil has a spectrum with circular support. ${ }^{3}$ Nyquist samples from such images are thus dependent, and lost samples can be evaluated from those remaining.

In this paper, after a brief review of the sampling theorem in $N$ dimensions, we derive specific formulas for restoring lost samples in certain Nyqust sampled signals. The sensitivity of the restoration to additive noise is then presented. The results are fascinating interpretations of noise levels based on areas of regions of support. (Here and later, area refers to $N$-dimensional area; e.g., for $N=3$, area refers to a volume). Applications to optical images are then addressed specifically.

\section{PRELIMINARIES}

Before stating the closed-form algorithm for lost-sample restoration, it is necessary to state the results of the $N$ dimensional sampling theorem for nonrectangular sampling geometry. Details of the theorem are admirably presented by Dudgeon and Mersereau4 from Petersen and Middleton's initial treatment. ${ }^{5}$

\section{$N$-Dimensional Sampling}

Let $\left\{x(\mathbf{t}) \mid \mathbf{t}=\left(t_{1}, t_{2}, \ldots, t_{N}\right)^{\prime}\right\}$ denote an $N$-dimensional signal. (The prime is for vector or matrix transposition.) The corresponding spectrum is

$$
X(\Omega)=\int_{t} x(\mathbf{t}) \exp \left(-j \Omega^{\prime} \mathrm{t}\right) \mathrm{d} \mathbf{t},
$$

where $\Omega=\left(\Omega_{1}, \Omega_{2}, \ldots, \Omega_{N}\right)^{\prime}$ and

$$
\int_{\mathrm{t}}=\int_{t_{1}} \int_{t_{2}} \ldots \int_{t_{N}} \text {. }
$$

The inverse transform is

$$
x(\mathbf{t})=\frac{1}{(2 \pi)^{N}} \int_{\Omega} X(\Omega) \exp \left(j \Omega^{\prime} \mathrm{t}\right) \mathrm{d} \Omega .
$$

Let $\mathbf{v}$ be an $N \times N$ sampling matrix corresponding to the manner in which $x(\mathbf{t})$ is sampled. In general,

$$
\mathbf{v}=\left[\mathbf{v}_{1}^{i} \mathbf{v}_{2} \ldots: \mathbf{v}_{N}\right],
$$

where the $\mathbf{v}_{n}$ 's are sampling vectors. For example, in Fig. 1, $N=2$ and

$$
\mathbf{v}=\left[\begin{array}{rr}
-1 & 2 \\
3 & -2
\end{array}\right]
$$

In general, the sampling density is

$$
D=\frac{1}{|\operatorname{det} \mathrm{V}|} \frac{\text { samples }}{\text { (unit length) }^{N}} \text {. }
$$

For a specified $\mathbf{v}$, the sample signal is

$$
\hat{x}(\mathbf{t})=\sum_{\mathbf{n}} x(\mathbf{V n}) \delta_{D}(\mathbf{t}-\mathbf{V n}),
$$




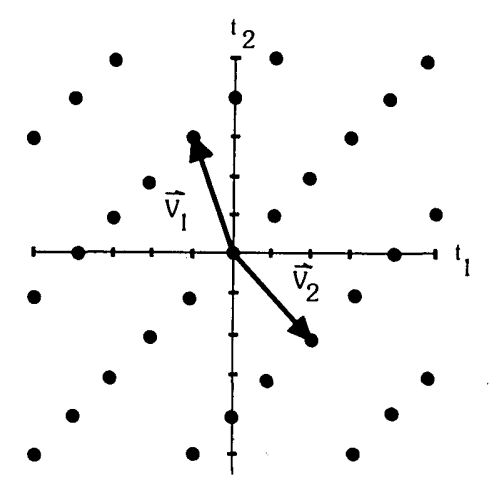

Fig. 1. Sampling geometry corresponding to the sampling matrix in Eq. (1).

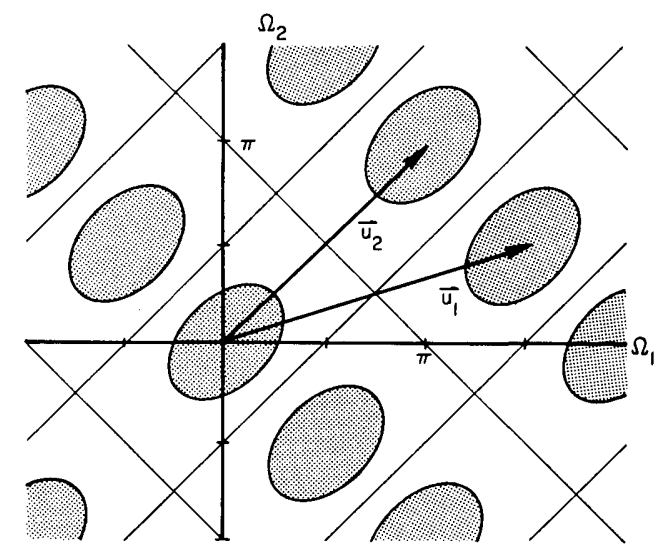

Fig. 2. Spectrum replication from the sampling geometry of Fig. 1.

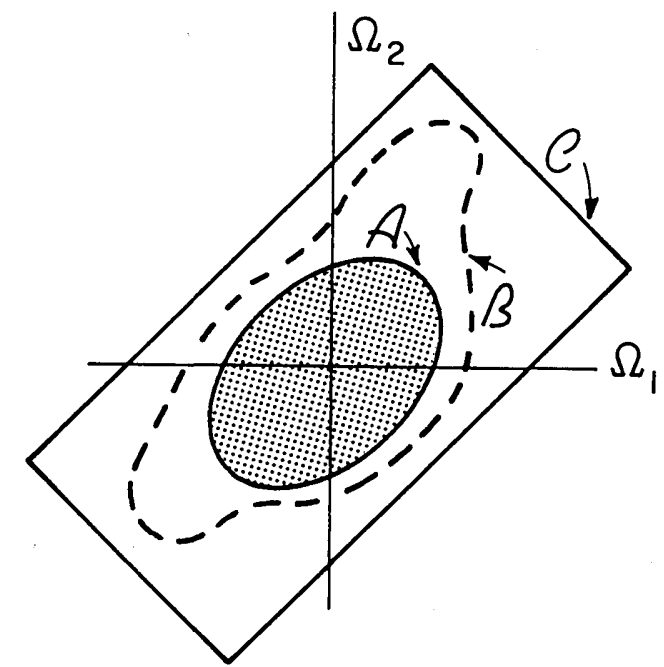

Fig. 3. One cell of Fig. 2. The region of integration, $\mathcal{B}$, must contain the spectral support region, $\mathcal{A}$, and must not infringe onto adjacent spectra. $\mathcal{C}$ is a cell region. The areas of the regions $\mathcal{A}, \mathcal{B}$, and $C$ are $A, B$, and $C$, respectively.

where $\delta_{D}(\cdot)$ is the Dirac delta and $\mathbf{n}=\left(n_{1}, \mathrm{n}_{2}, \ldots, n_{N}\right)^{\prime}$. The spectrum of $\hat{x}(\mathrm{t})$ is the replication of the spectrum of $x(t)$ :

$$
\hat{X}(\Omega)=D \sum_{k} X(\Omega-\mathbf{u k})
$$

where $\mathbf{U}$, the Fourier periodicity matrix, satisfies

$$
\mathbf{U}^{\prime} \mathbf{V}=2 \pi \mathbf{I} \text {. }
$$

As we shall see, the geometry of the replication is dictated by $\left\{\mathbf{u}_{n} \mid n=1,2, \ldots, N\right\}$, where

$$
\mathbf{u}=\left[\mathbf{u}_{1} \mathfrak{i} \mathbf{u}_{2}^{i} \ldots \mid \mathbf{u}_{N}\right]
$$

For our example in Eq. (1),

$$
\mathbf{U}=\left[\begin{array}{rr}
\pi & 3 \pi / 2 \\
\pi & \pi / 2
\end{array}\right] .
$$

Thus, if $X\left(\Omega_{1}, \Omega_{2}\right)$ were confined to be within the shaded ellipse at the origin in Fig. 2, then the corresponding $\hat{X}\left(\Omega_{1}\right.$, $\Omega_{2}$ ) would have the periodic structure shown.

For a given $\mathbf{v}$, there can exist a number of ways to separate $\hat{X}(\Omega)$ into periods. A period cell, when replicated, must fill the entire $\Omega$ plane. For a given $\mathbf{v}$, all cells will clearly have the same area. A possible cell for the example in Fig. 2 is the rotated rectangle shown in Fig. 3.

\section{The $N$-Dimensional Sampling Theorem}

An $N$-dimensional signal is band limited in the low-pass sense if its spectrum is identically zero outside of an $N$ dimensional hypersphere of finite radius. Then we can find a sampling matrix $\mathbf{V}$ such that the corresponding sample spectrum consists of nonoverlapping components. Under this condition, it is possible to regain $X(\Omega)$ from $\hat{X}(\Omega)$ in Eq. (3). We choose a region $\mathcal{B} \in \Omega$ that contains only the zerothorder spectrum. Then

$$
X(\Omega)=\hat{X}(\Omega) F(\Omega),
$$

where

$$
F(\Omega)=\left\{\begin{array}{cc}
|\operatorname{det} v| ; & \Omega \in \mathcal{B} \\
0 ; & \Omega \notin \mathcal{B}
\end{array} .\right.
$$

An illustration for our running example is shown in Fig. 3. Note that $\mathcal{B}$ could correspond to a cell region $\mathcal{C}$ or the spectrum's region of support $\mathcal{A}$. To regain $x(\mathbf{t})$, we inverse transform Eq. (5) and obtain

$$
x(\mathbf{t})=\hat{x}(\mathbf{t}) * f(\mathbf{t}),
$$

where the asterisk denotes $N$-dimensional convolution and

$$
f(\mathbf{t})=\frac{|\operatorname{det} \mathbf{V}|}{(2 \pi)^{N}} \int_{\mathcal{B}} \exp \left(j \Omega^{\prime} \mathbf{t}\right) \mathrm{d} \Omega .
$$

Substituting Eq. (2) gives the desired interpolation formula:

$$
x(\mathbf{t})=\sum_{\mathbf{n}} x(\mathbf{V n}) f(\mathbf{t}-\mathbf{V n}) .
$$

\section{RESTORING LOST SAMPLES}

In this section, we will show that an arbitrarily large but finite number of lost samples can be regained from those remaining for certain band-limited signals even when sampling is performed at the minimum density. The problem addressed is one of well-posed interpolation rather than illposed extrapolation. ${ }^{6-9}$

Let $M$ denote a set of $M$ integer vectors corresponding to the $M$ lost-sample locations in an $N$-dimensional band-limited signal sampled in accordance with a sampling matrix, $\mathbf{V}$.

Theorem: If $x(\mathbf{t})$ is a band-limited signal and $\mathbf{v}$ is chosen to ensure that there is no aliasing between adjacent cells, 
then the missing samples can be regained from solution of the $M$ equations:

$\sum_{\mathbf{n} \in \mathcal{M}}\{\delta(\mathbf{k}-\mathbf{n})-f[\mathbf{v}(\mathbf{k}-\mathbf{n})]\} x(\mathbf{V n})=\sum_{\mathbf{n} \notin M} x(\mathbf{V n}) f[\mathbf{v}(\mathbf{k}-\mathbf{n})]$

$\mathbf{k} \in M$

assuming that the solution is not singular. [The Kronecker delta function, $\delta(\mathbf{n})$, is unity when $\mathbf{n}=\mathbf{O}$ and is zero otherwise.] The left-hand side of Eq. (8) contains the unknown samples. The right-hand side can be found from the known data. 1 ,

Corollary: For a single lost sample at the origin, if $f(\mathbf{O}) \neq$

$$
x(\mathbf{O})=[1-f(\mathbf{O})]^{-1} \sum_{\mathbf{n} \neq \mathbf{0}} x(\mathbf{V n}) f(-\mathbf{V n}) .
$$

This follows from Eq. (8) for $M=1$ and $M$ containing only the origin. Note that, by using Eq. (7), the signal's interpolation can be written directly void of the sample at the origin:

$$
x(\mathbf{t})=\sum_{\mathbf{n} \neq \mathbf{0}} x(\mathbf{V n})\left[f(t-\mathbf{V n})+\{1-f(\mathbf{O})\}^{-1} f(-\mathbf{V n}) f(\mathbf{t})\right] .
$$

Theorem Proof: We can write Eq. (7) as

$$
x(\mathbf{t})=\left(\sum_{\mathbf{n} \in \mathcal{M}}+\sum_{\mathbf{n} \notin \mathcal{M}}\right) x(\mathbf{V n}) f(\mathbf{t}-\mathbf{V n}) .
$$

This expression can be evaluated at $M$ points, and we can solve for $\{x(\mathbf{V n}) \mid \mathbf{n} \in M\}$. Let these $M$ points be the $\mathbf{t}=\mathbf{v k}$, where $\mathbf{k} \in \mathcal{M}$ :

$$
x(\mathbf{v k})=\left(\sum_{\mathbf{n} \in M}+\sum_{\mathbf{n} \notin \mathcal{M}}\right) x(\mathbf{V n}) f\{\mathbf{v}(\mathbf{k}-\mathbf{n})\} ; \quad \mathbf{k} \in M .
$$

Rearranging gives Eq. (8).

Corollary: A sufficient condition for Eq. (8) to be singular is when the integration region, $\mathcal{B}$, is equal to a cell region, $\mathcal{C}$.

Proof: On a cell, the functions $\left\{\exp \left(j \Omega^{\prime} \mathbf{V n}\right)\right\}$ form an orthogonal basis set. From Eq. (6) with $\mathcal{B}=\mathcal{C}$ we have

$$
\begin{aligned}
f(\mathbf{V n}) & =\frac{|\operatorname{det} \mathbf{V}|}{(2 \pi)^{N}} \int_{\mathcal{C}} \exp \left(j \Omega^{\prime} \mathbf{V} \mathbf{n}\right) \mathrm{d} \Omega . \\
& =\delta(\mathbf{n}) .
\end{aligned}
$$

The left-hand side of Eq. (8) is thus zero and the resulting set of equations singular.

The restoration algorithm in this section alternatively could have been derived by a generalization of the iterative technique in Ref. 1. The treatment here, however, is more compact although maybe less intuitive. The results in Ref. 1 are equivalent to the $N=1$ case. The same is true of Section 4 and Ref. 2.

\section{NOISE SENSITIVITY}

Our purpose here is to investigate the restoration algorithm's performance when inaccurate data are used. ${ }^{2,10}$ In general, the algorithm becomes more unstable when (1) $M$ increases and/or (2) the area corresponding to $\mathcal{B}$ increases with respect to that of $\mathcal{C}$. Indeed, restoration is no longer possible when $\mathcal{B}=\mathcal{C}$.

The restoration algorithm in Eq. (8) is linear. Let $\xi(\mathbf{t})$ denote a zero mean stochastic process. If $x(\mathrm{t})$ is uncorrelated with $\xi(\mathbf{t})$, then the use of $\{x(\mathbf{V n})+\xi(\mathbf{V n}) \mid \mathbf{n} \notin \mathcal{M}\}$ in Eq. (8) instead of $\{x(\mathbf{V n}) \mid \mathbf{n} \notin \mathcal{M}\}$ will result in $\{x(\mathbf{V n})+\eta(\mathbf{V n}) \mid \mathbf{n} \in \mathcal{M}\}$, where $\{\eta(\mathbf{V n}) \mid \mathbf{n} \in \mathcal{M}\}$ is the response to $\{\xi(\mathbf{V n}) \mid \mathbf{n} \notin \mathcal{M}\}$ alone:

$$
\sum_{\mathbf{n} \in \mathcal{M}}[\delta(\mathbf{k}-\mathbf{n})-f\{\mathbf{v}(\mathbf{k}-\mathbf{n})\}] \eta(\mathbf{V n})=\sum_{\mathbf{n} \notin \mathcal{M}} \xi(\mathbf{V n}) f\{\mathbf{v}(\mathbf{k}-\mathbf{n})\} .
$$

The restoration noise, $\eta$, depends linearly on the data noise, $\xi$. Thus the cross correlation between these two processes and the autocorrelation of $\eta$ can be determined from a given data noise autocorrelation. ${ }^{11}$

Out treatment will be limited to the case when a single sample is lost and the data noise is samplewise white, i.e.,

$$
E\left[\xi(\mathbf{V n}) \xi^{*}(\mathbf{V m})\right]=\overline{\xi^{2}} \delta(\mathbf{n}-\mathbf{m}),
$$

where $\overline{\xi^{2}}$ is the data noise level (variance) and $E$ denotes expectation. With no loss in generality, we place the lost sample at the origin, and Eq. (10) becomes

$$
\eta(\mathbf{O})=[1-f(\mathbf{O})]^{-1} \sum_{\mathbf{n} \neq \mathbf{0}} \xi(\mathbf{V n}) f(-\mathbf{V n}) .
$$

Taking the square of the magnitude, expectating, and using Eq. (11) gives

$$
\overline{\eta^{2}(\mathbf{O})} / \overline{\xi^{2}}=[1-f(\mathbf{O})]^{-2} \sum_{\mathbf{n} \neq \mathbf{0}}|f(-\mathbf{V n})|^{2},
$$

where the restoration noise level is

$$
\overline{\eta^{2}(\mathbf{O})}=E\left[|\eta(\mathbf{O})|^{2}\right] \text {. }
$$

The sum in Eq. (12) can be evaluated through Eq. (9) with $x(\mathbf{t})=f^{*}(-t)[=f(t)$ since $F(\Omega)$ is real $]$. The result is

$$
\overline{\eta^{2}(\mathbf{O})} / \overline{\xi^{2}}=\frac{f(\mathbf{O})}{1-f(\mathbf{O})} .
$$

The result has a fascinating geometrical interpretation. From Eq. (6)

$$
f(\mathbf{O})=\frac{|\operatorname{det} \mathrm{V}|}{(2 \pi)^{N}} \int_{\mathcal{B}} \mathrm{d} \Omega .
$$

But, with an illustration in Fig. 3,

$$
\begin{aligned}
B & =\int_{\mathcal{B}} \mathrm{d} \Omega \\
& =\text { area of integration, } \mathscr{B}
\end{aligned}
$$

and

$$
\begin{aligned}
C & =\int_{\mathcal{C}} \mathrm{d} \Omega \\
& =\text { area of cell, } \mathcal{C} \\
& =|\operatorname{det} \mathrm{U}| \\
& =(2 \pi)^{N} /|\operatorname{det} \mathrm{V}|,
\end{aligned}
$$

where we have used Eq. (4). Thus Eq. (13) can be written as 


$$
\frac{\overline{\eta^{2}(\mathbf{O})}}{\xi^{2}}=\left(\frac{C}{B}-1\right)^{-1} .
$$

The restoration noise level is thus directly determined by the areas of the integration region for $f(t)$ and the area of a cell. Equation (14) is a strictly increasing function of $B$. Thus, for minimum restoration noise level, we choose $\mathcal{B}=\mathcal{A}$ $=$ the region of support of the signal $x(t)$.

For Nyquist density sampling in one dimension, $\mathcal{A}=\mathcal{B}=$ c. In this case oversampling is required to restore lost samples. ${ }^{1}$ For higher dimensions, the restoration capability is dependent on the region of support of the signal's spectrum. If the support is the shape of a cell (e.g., rectangular, hexagonal), then restoration is not possible at the Nyquist density.

\section{Filtering}

Samplewise white noise has a uniform spectral density and thus significant high-frequency energy. Once lost data have been restored, the data noise level can be reduced by filtering the result through $\mathscr{B}$ assuming that $B<C$. The noise level at the lost sample location remains the same. ${ }^{2}$ The noise level at locations far removed from the lost-sample locations will asymptotically be the same as that for the filtered noisy samples if no data were lost. If $\xi(\mathbf{V} n)$ is zero mean and stationary, then after filtering, the process $\psi(\mathbf{V} n)$ is also stationary. If the data noise is white as in Eq. (11), its spectral density is uniform in $\mathcal{C}$. Thus if we filter the noise through $\mathcal{B}$, the resulting normalized noise level is

$$
\overline{\psi^{2}} / \overline{\xi^{2}}=B / C \text {. }
$$

(A more rigorous derivation is given in Appendix A.) To minimize, we clearly would choose $\mathcal{B}=\mathcal{A}$.

For a single lost sample in samplewise white noise, the ratio of the restoration noise level to that of data far removed is, after filtering through $\mathcal{B}$,

$$
\overline{\frac{\eta^{2}(\mathbf{O})}{\overline{\psi^{2}}}}=\left[1-\frac{B}{C}\right]^{-1},
$$

where we have used Eqs. (14) and (15). To minimize, we again would choose $\mathscr{B}=\mathcal{A}$. Note that Eq. (16) exceeds both unity and Eq. (14).

\section{APPLICATION TO IMAGING SYSTEMS}

An object of finite extent is imaged through a system with a circular pupil. If the monochromatic illumination is either coherent or incoherent, the image will have a spectrum with support inside a circle whose radius $W$ is proportional to that of the pupil.

\section{Nyquist Sampling of Optical Images}

The Nyquist sampling density here is achieved when the circles in the frequency domain are densely packed as is shown at the top of Fig. 4. This corresponds to a sampling matrix

$$
\mathbf{v}=\left[\begin{array}{cc}
T & -T \\
T / \sqrt{3} & T / \sqrt{3}
\end{array}\right],
$$

where $T=\pi / W$. The corresponding optimal sampling geometry, shown in Fig. 5, is thus hexagonal. ${ }^{4}$
Note, as is shown at the bottom of Fig. 4, that the area of $\mathcal{A}$ is less than that of $\mathcal{C}$. Thus, in the absence of noise, an arbitrary number of lost image samples can be restored from those (infinite number) remaining. For $\mathcal{B}=\mathcal{A}$, the interpolation function here is ${ }^{3}$

$$
f\left(t_{1}, t_{2}\right)=\frac{W}{2 \pi D} \frac{J_{1}\left[W\left(t_{1}{ }^{2}+t_{2}{ }^{2}\right)^{1 / 2}\right]}{\left(t_{1}{ }^{2}+t_{2}{ }^{2}\right)^{1 / 2}} .
$$

\section{Noise Effects}

Here, we will numerically illustrate the effects of samplewise white noise on restoring a lost sample from an image that has a spectrum with circular support. Suboptimal rectangular sampling is considered first, followed by the optimal hexagonal case. Both cases are extended to higher dimensions.
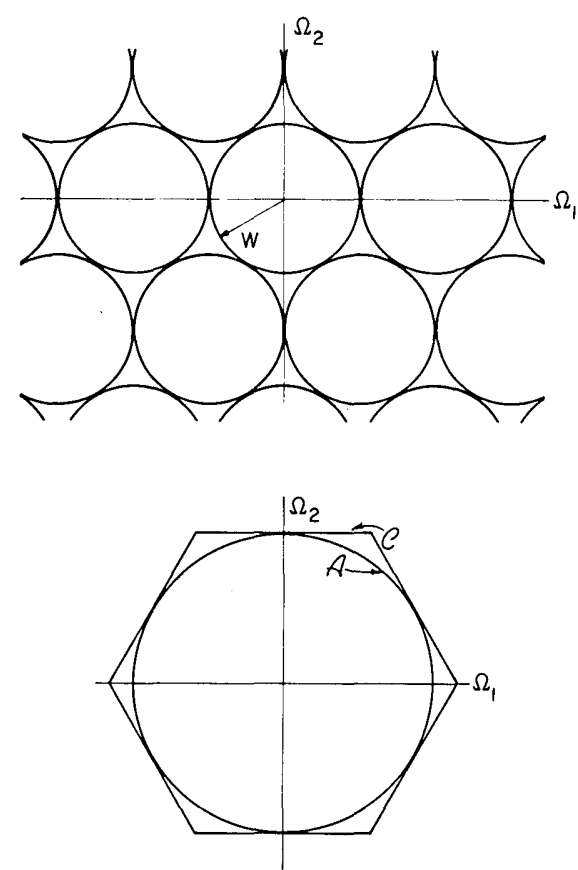

Fig. 4. Top, densely packed circles correspond to Nyquist sampling of images with spectra of circular support. Note the hexagonal structure. Bottom, a single hexagonal cell with inscribed circular spectrum support.

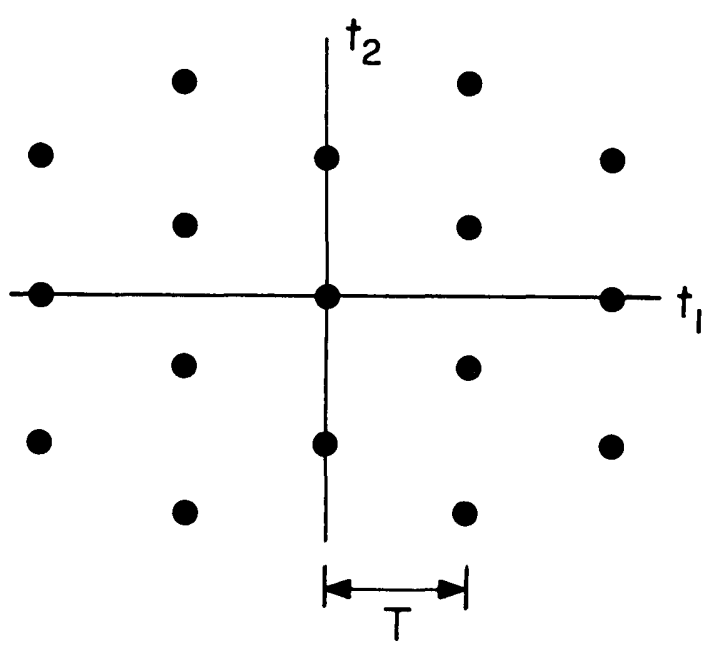

Fig. 5. Hexagonal sampling geometry required to pack circles densely as shown in Fig. 4. 


\section{Rectangular Sampling}

If limited to rectangular sampling, minimum density sampling is accomplished by the sampling matrix

$$
\mathbf{v}=\left[\begin{array}{cc}
T & 0 \\
0 & T
\end{array}\right]
$$

where $T=\pi / W$. The corresponding replicated spectra are shown at the top of Fig. 6 . A single cell of this replication is shown on the bottom. The restoration noise level from Eq. (14) follows as
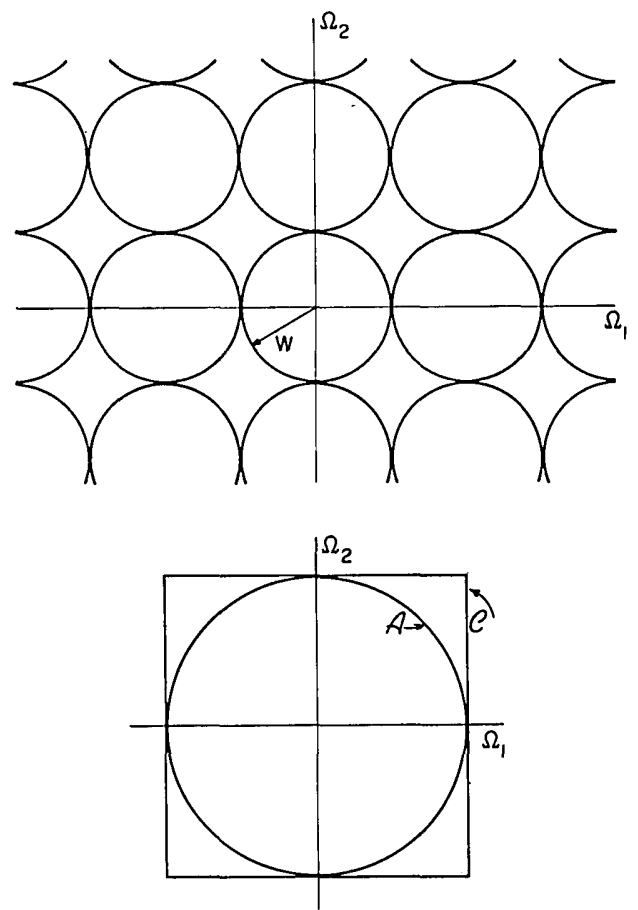

Fig. 6. Top, minimum density rectangular sampling of images with spectra of circular support yields circles packed as shown. Bottom, a single cell with inscribed circular spectrum support.

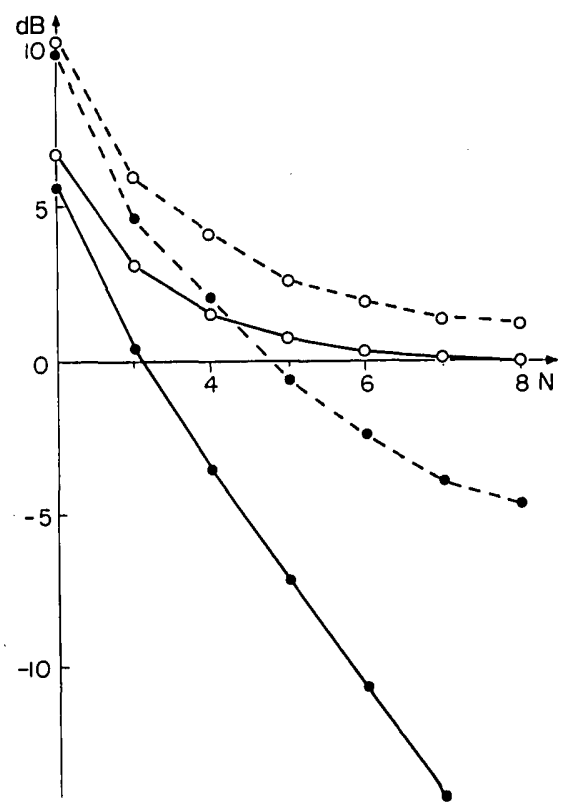

Fig. 7. Plots of $\overline{\eta^{2}(0)} / \overline{\xi^{2}}$ (filled circles) and $\overline{\eta^{2}(0)} / \overline{\psi^{2}}$ (open circles) in $\mathrm{dB}\left[10 \log _{10}(\cdot)\right]$. The solid lines are for minimum density rectangular sampling and the dashed for Nyquist (hexagonal) sampling.

$$
\begin{aligned}
\frac{\overline{\eta^{2}(\mathbf{O})}}{\overline{\xi^{2}}} & =\left(\frac{4}{\pi}-1\right)^{-1} . \\
& \simeq 3.66
\end{aligned}
$$

After filtering through the $\mathcal{A}$ circle, the ratio of the restoration noise level to data at points far removed from the origin is

$$
\begin{aligned}
\frac{\overline{\eta^{2}(\mathbf{O})}}{\overline{\psi^{2}}} & =\left[1-\frac{\pi}{4}\right]^{-1}, \\
& \simeq 4.66
\end{aligned}
$$

where we have used Eq. (16) with $B=A=\pi W^{2}$. The lostsample noise is thus $6.7 \mathrm{~dB}$ above the filtered data noise at infinity.

The results can easily be extended to higher dimensions. Assume that the spectrum has support within an $N$-dimensional hypersphere of radius $W$ (Ref. 12):

$$
A=\left\{\begin{array}{ll}
\frac{2^{N} \pi^{(N-1) / 2}\left(\frac{N-1}{2}\right) ! W^{N}}{N !} & \operatorname{odd} N \\
\frac{\pi^{N / 2}}{\left(\frac{N}{2}\right) !} W^{N} & \text { even } N
\end{array} .\right.
$$

For rectangular sampling, $C=(2 W)^{N}$. The corresponding plots of $\overline{\eta^{2}(0)} / \overline{\xi^{2}}$ and $\overline{\eta^{2}(0)} / \overline{\psi^{2}}$ are shown as solid lines in Fig. 7.

\section{Hexagonal Sampling}

A single hexagonal cell is shown at the bottom of Fig. 7 for minimum density sampling. The area of the hexagon is

$$
C=2 \sqrt{3} W^{2} \text {. }
$$

Thus, from Eq. (14) for $B=\mathrm{A}=\pi W^{2}$

$$
\begin{aligned}
\frac{\overline{\eta^{2}(\mathbf{O})}}{\overline{\xi^{2}}} & =\left(\frac{2 \sqrt{3}}{\pi}-1\right)^{-1} \\
& \simeq 9.74,
\end{aligned}
$$

and, similarly, from Eq. (16)

$$
\begin{aligned}
\frac{\overline{\eta^{2}(\mathbf{O})}}{\overline{\psi^{2}}} & =\left(1-\frac{\pi}{2 \sqrt{3}}\right)^{-1} . \\
& \simeq 10.74
\end{aligned}
$$

As one would expect, these values $(\sim 10 \mathrm{~dB})$ are greater than those of the corresponding rectangular sampling cases in Eqs. (17) and (18).

In higher dimensions, Nyquist sampling would correspond to densely packed hyperspheres in the frequency domain. A table of the cell volume to circumscribed cubic volume is given by Dudgeon and Mersereau. ${ }^{4}$ We can use this table in conjunction with Eq. (19) to generate the restoration noise level plots in Fig. 7 for Nyquist density sampling when the signal's spectrum support is a hypersphere. The plots are shown with broken lines and, as we would expect, exceed the corresponding rectangular sampling results.

\section{CONCLUSIONS}

We have shown that, in the absence of noise, an arbitrarily large but finite number of lost samples can be regained from 
those samples remaining under the conditions that (a) the data (with the lost samples) are not aliased and (b) there are sections in the sampled signal's spectrum that are identically zero. In dimensions greater than one, these conditions can apply even at Nyquist densities.

Noise analysis was performed for the case of one lost sample when the remaining data were corrupted by zero mean stationary white noise in terms of the sample. The resulting restoration noise levels are given by simple algebraic expressions involving various areas in the frequency domain. In all cases, minimum restoration noise level was achieved when the area of the support of the interpolation function's spectrum was at its minimum allowable value.

\section{APPENDIX A}

Here we derive Eq. (15). Let the samples be subjected to noise, $\xi(\mathbf{V n})$, with autocorrelation as in Eq. (11). Then if $x(\mathbf{V n})+\xi(\mathbf{V n})$ is used in Eq. (7) in lieu of $x(\mathbf{V n})$, the result is $x(\mathbf{t})+\psi(\mathbf{t})$, where

$$
\psi(\mathbf{t})=\sum_{\mathbf{n}} \xi(\mathbf{V n}) f(\mathbf{t}-\mathbf{V n}) .
$$

Squaring the magnitude of both sides and taking the expected value gives

$$
\overline{\psi^{2}(\mathbf{t})}=\overline{\xi^{2}} \sum_{\mathbf{n}}|f(\mathbf{t}-\mathbf{v n})|^{2} .
$$

This sum can be evaluated using Eq. (7) with $x(\mathbf{t})=f^{*}(\tau-$ t):

$$
f^{*}(\boldsymbol{\tau}-\mathbf{t})=\sum_{\mathbf{n}} f^{*}(\boldsymbol{\tau}-\mathbf{V n}) f(\mathbf{t}-\mathbf{V n})
$$

For $\tau=\mathbf{t}$ we obtain Eq. (15), recognizing that $\psi^{2}(\mathbf{t})=\psi^{2}$ is independent of $\mathbf{t}$.

Note that this result is a quantitative mesure of the tradeoff between sampling density and interpolation noise level.

\section{ACKNOWLEDGMENTS}

The author expresses his appreciation to Kwan F. Cheung for some valuable points and to Loretta Tollefson and Heidi Nurk for their assistance with the transcript and figures. Both reviewers kindly pointed out some further important references included here. One reviewer suggested the compact proof of the second corollary in Section 3, which was far superior to the author's initial effort.

\section{REFERENCES}

1. R. J. Marks II, "Restoring lost samples from an oversampled bandlimited signal," IEEE Trans. Acoust. Speech Signal Process. ASSP-31, 752-755 (1983).

2. R. J. Marks II and D. Radbel, "Error of linear estimation of lost samples in an oversampled band-limited signal," IEEE Trans. Acoust. Speech Signal Process. ASSP-32, 648-654 (1984).

3. J. W. Goodman, Introduction to Fourier Optics (McGraw-Hill, New York, 1968).

4. D. E. Dudgeon and R. M. Mersereau, Multidimensional Digital Signal Processing, (Prentice-Hall, Englewood Cliffs, N.J., 1984), Chap. 1.

5. D. P. Peterson and D. Middleton, "Sampling and reconstruction of wave-number-limited function in $N$-dimensional Euclidean spaces," Inf. Control 5, 279-323 (1962).

6. A. K. Jain and S. Ranganath, "Extrapolation algorithms for discrete signals with application in spectral estimation," IEEE Trans. Acoust. Speech Signal Process, ASSP-29, 830-845 (1981).

7. K. M. Rege, "Min-max linear estimation of band-limited sequences from noisy observations: a deterministic approach," IEEE Trans. Inf. Theory IT-29, 902-909 (1983).

8. J. L. C. Sanz and T.S. Huang, "A unified approach to noniterative linear signal restoration," IEEE Trans. Acoust. Speech Signal Process, ASSP-32, 403-409 (1984).

9. R. J. Marks II and D. K. Smith, "Gerchberg-type linear deconvolution and extrapolation algorithms," Proc. Soc. Photo-Opt. Instrument. Eng. 373, 161-178 (1984).

10. A. A. Melkman and C. A. Micchelli, "Optical estimation of linear operators in Hilbert spaces from inaccurate data," SIAM J. Numer. Anal. 16, 87-105 (1979).

11. A. Papoulis, Probability, Random Variables and Stochastic Processes, 2nd ed. (McGraw-Hill, New York, 1984), pp. 235-237.

12. J. Wozencraft and I. M. Jacobs, Principles of Communication Engineering (Wiley, New York, 1965), pp. 355-357. 